\title{
Coexistence of Gastric Adenocarcinoma and Choriocarcinoma: Complete Response to Trastuzumab and Chemotherapy
}

\author{
Seyda Gunduz ${ }^{a} \quad$ Gulsum Ozlem Elpek $^{\mathrm{b}} \quad$ Mukremin Uysal $^{\mathrm{a}}$ \\ Sema Sezgin Goksu ${ }^{a}$ Murat Tatlı ${ }^{a}$ Deniz Arslan ${ }^{a}$ \\ Hasan Senol Coşkun ${ }^{a}$ Hakan Bozcuk $^{a}$ Burhan Savas ${ }^{a}$ \\ Mustafa Ozdogan ${ }^{a}$
}

Departments of ${ }^{\mathrm{a}}$ Medical Oncology and ${ }^{\mathrm{b}}$ Pathology, Akdeniz University Faculty

of Medicine, Antalya, Turkey

\section{Key Words}

Gastric choriocarcinoma $\cdot$ cErbB2 $\cdot$ Trastuzumab

\begin{abstract}
Gastric choriocarcinoma is a rare neoplasm and usually accompanies gastric adenocarcinoma. The prognosis is poor due to the aggressive course of the disease. A 57year-old female patient with weight loss and abdominal pain was examined. The patient was operated following the examination, and pathological analysis revealed the presence of a gastric adenocarcinoma associated with choriocarcinoma. Immunohistochemical analysis showed a positive reaction with antibodies to beta-human chorionic gonadotropin and overexpression of the cErbB2 proto-oncogene. Staging revealed multiple metastases in the liver. A complete response was obtained with a combination of trastuzumab and chemotherapy. The diagnosis of gastric choriocarcinomas without pathological examination is difficult due to their rare occurrence. A complete response can be obtained with trastuzumab in the treatment of cases with overexpression of the CErbB2 protein.
\end{abstract}

\section{Introduction}

Choriocarcinomas are generally gestational and have an intrauterine location. Nongestational choriocarcinomas are rarely seen outside the gonads, with the mediastinum 
being the main site for these non-gestational extragonadal choriocarcinomas. Primary gastric choriocarcinoma is extremely rare. This neoplasm grows very quickly and has a poor prognosis. There is no consensus regarding the use of systemic chemotherapy [1, 2]. Studies undertaken on treatment for the metastatic disease indicate that chemotherapies based on cisplatin, methotrexate or 5-FU are not efficient [1,2]. There is no metastatic gastric choriocarcinoma case in the literature that showed a complete response to chemotherapy. Furthermore, there is no information concerning trastuzumab treatment. Herein, we present a Human Epidermal growth factor Receptor-2 (HER2)-positive gastric adenocarcinoma case which has a choriocarcinomatous component and showed a complete response to trastuzumab chemotherapy.

\section{Case Report}

A 57-year-old female patient was admitted for weakness, lack of appetite, weight loss and abdominal pain. There was no accompanying melena, nausea and vomiting. The patient did not have a disease history and had not been under any medical treatment. On physical examination the mucosa was pale and no abdominal mass was detected. Laboratory analysis revealed hypochromic, microcytic anemia (Hb: $9 \mathrm{~g} / \mathrm{dl}$ ). Upper endoscopic examination revealed a mass in the gastric antrum, which was reported to be adenocarcinoma by biopsy. Abdominal computed tomography (CT) and chest radiography revealed no mass. The patient underwent subtotal gastrectomy. Postoperative pathological analysis revealed the presence of adenocarcinoma associated with choriocarcinoma; histologically, intestinal type adenocarcinoma composed of columnar gland-forming cells interspersed with cytotrophoblast and syncytiotrophoblast cells staining positive for beta-human chorionic gonadotropin ( $\beta$-HCG) on immunohistochemistry ( fig. 1, fig. 2 ). The patient's serum $\beta$-HCG level, which was measured in light of these pathological findings, was $22.823 \mathrm{IU} / \mathrm{ml}$. Multiple liver metastases and a metastasis in the right iliac bone were detected on PET-CT imaging for tumor staging. The patient was given two cycles of DCF (docetaxel, cisplatin, 5-FU) as treatment. However, the patient did not tolerate the treatment due to the development of grade 3 diarrhea and mucositis. Immunohistochemical staining of pathological specimens showed HER2 overexpression. Therefore, the DCF treatment was replaced with a combination of docetaxel, carboplatin and trastuzumab. The patient's clinical condition improved significantly after two cycles of treatment. $\beta$-HCG level had decreased to $6 \mathrm{IU} / \mathrm{ml}$, and PET-CT scans showed a complete response (fig. 3). After six cycles of the initial dose, maintenance therapy was continued with a combination of trastuzumab and capecitabine. The patient is currently on the 24 th month of the treatment and the complete response is still ongoing.

\section{Discussion}

Gastric choriocarcinoma is extremely rare and forms $0.08 \%$ of all gastric cancers [1]. This form of cancer was described for the first time in 1905 by Davidsohn [3]. There are some theories concerning the pathogenesis of the disease. The most widely accepted theory holds that the trophoblastic cells in gastric choriocarcinoma develop from dedifferentiation of a poorly differentiated adenocarcinoma [4]. In fact, cytogenetic studies show that gastric choriocarcinoma genetically possesses characteristics of both adenocarcinoma and choriocarcinoma [5]. In our patient choriocarcinoma accompanied adenocarcinoma and there was a gradual transition from adenocarcinoma to choriocarcinoma.

Clinical diagnosis of gastric choriocarcinoma is difficult due to its rare occurrence. Studies show that the diagnosis is established in most patients following a gastrectomy. 
Therefore, pathological analysis of the biopsy sample by an experienced pathologist increases the chance of establishing a diagnosis.

An elevation of serum $\beta$-HCG level is observed in patients with gastric choriocarcinoma. Consecutive measurements of serum $\beta$-HCG levels in the postoperative or post-chemotherapy periods are valuable for the assessment of treatment response or recurrence of the disease.

Most gastric choriocarcinoma patients have metastases at the time of establishing a diagnosis. The most common metastasis sites are lymph nodes (87\%), followed by liver (45\%), peritoneum (23\%) and lungs (8\%) [1]. Gastric choriocarcinomas are highly malignant and most patients die within a year of diagnosis. Although more than 140 gastric choriocarcinoma cases have been reported in the literature until now, there is no consensus concerning the treatment strategy for the disease [3]. In addition to surgery and radiotherapy, various cytotoxic drugs have been used for the treatment. However, contrary to gestational choriocarcinomas, all chemotherapy regimens were found to be ineffective in metastatic gastric choriocarcinoma [2]. A choriocarcinoma case who received trastuzumab therapy was not found in the literature.

In gastric cancers HER2 positivity is $25 \%$ and is associated with poor prognosis. However, HER2-positive gastric cancer patients benefit significantly from trastuzumab therapy [6]. When compared with normal placenta or HER2 expression is significantly stronger in choriocarcinoma $(\mathrm{p}<0.0001)$ [7]. Furthermore, choriocarcinoma components showed significantly higher HER2/neu expression compared to other histological subtypes in testicular germ cell tumors $(p=0.0095)$ [8]. These data suggest that cErbB2 oncoprotein may be important in the pathogenesis of choriocarcinoma [7], but there is no information concerning the use of trastuzumab for the treatment. HER2 overexpression was present in our case and complete response was obtained after adding trastuzumab to the treatment.

\section{Conclusion}

In order to establish a preoperative diagnosis for gastric choriocarcinoma, it is important to bear this tumor in mind and adopt a skeptical approach. Elevated serum $\beta$-HCG level is a clue for the diagnosis. Most patients have metastases at the time of establishing a diagnosis and the tumor has a significantly poor prognosis. Gastric choriocarcinoma is not as chemotherapy sensitive as pure choriocarcinomas. Determining HER2 expression in every patient and using trastuzumab treatment in positive cases may lead to positive therapeutic outcomes, as in our case.

\section{Disclosure Statement}

There is no conflict of interest. 

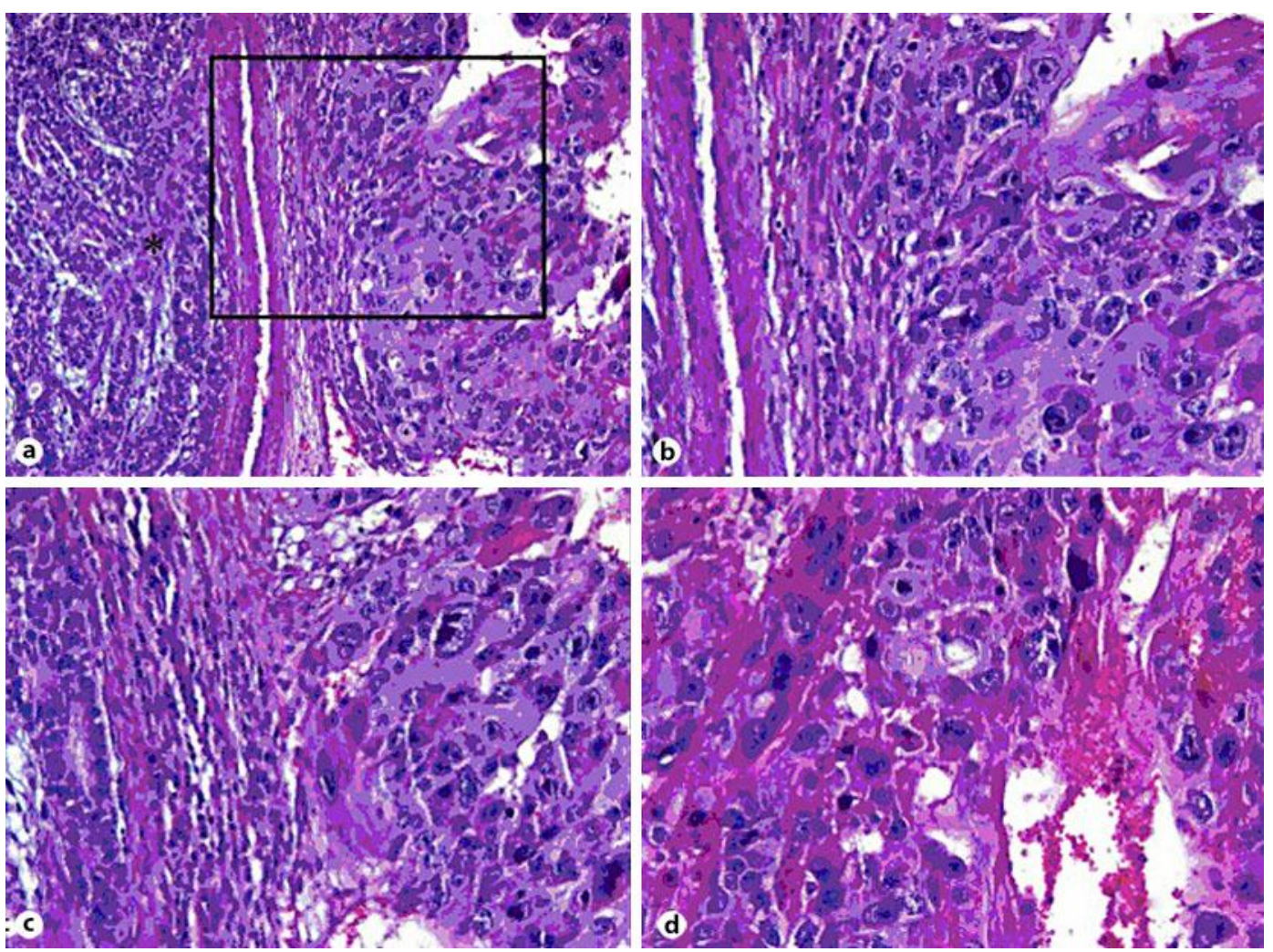

Fig. 1. a, b Gland-like structures that make up the tumor area and adjacent areas of choriocarcinoma. c, d Trophoblastic cells (HE, original magnification, $\times 100(a)$ and $\times 200(b, c, d)$ ). 

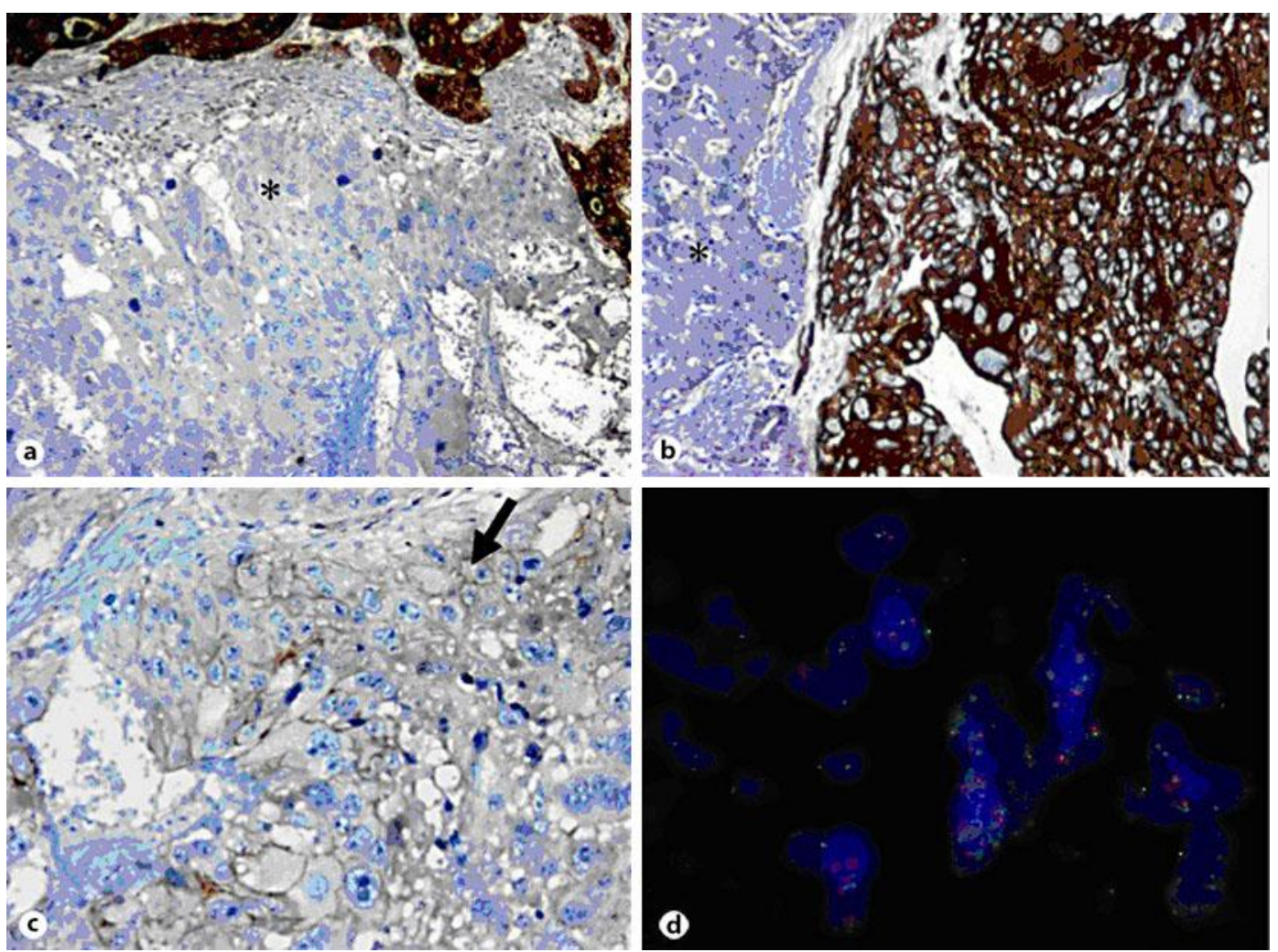

Fig. 2. a Polyclonal carcinoembryonic antigen is strongly positive in adenocarcinomatosis area. b Trophoblastic cells were strongly positive with antibodies to $\beta$-HCG. c Tumor cells showed positive reaction with HER-2. $d$ FISH is positive.
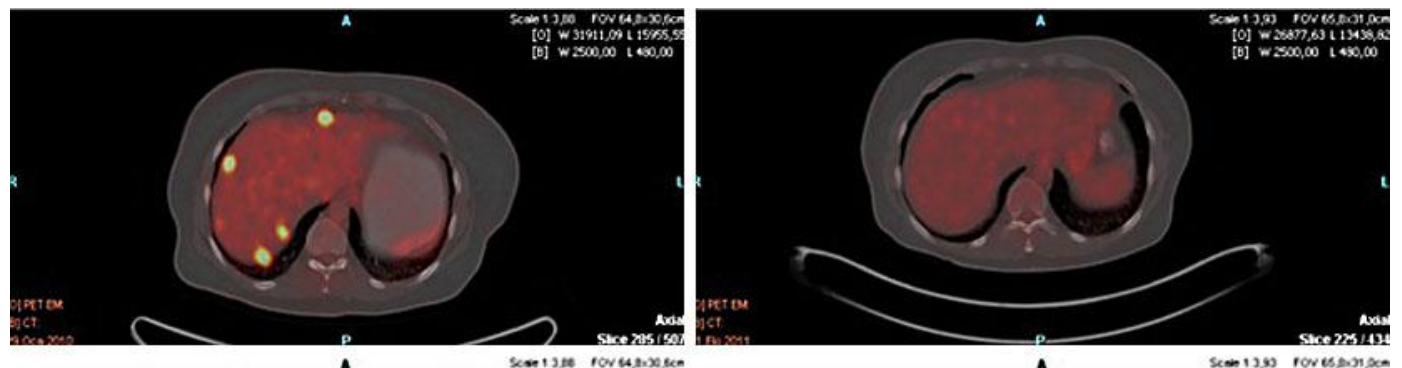

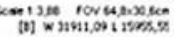

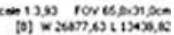
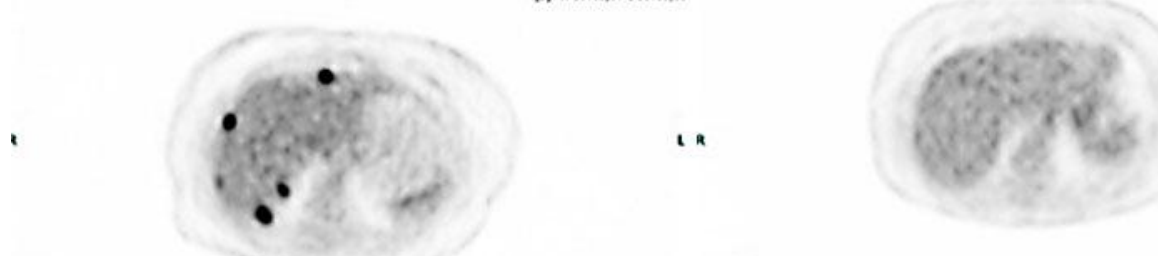

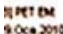

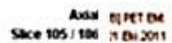

Sece 1 so ind

Fig. 3. Pretreatment and posttreatment. 


\section{References}

1 Kobayashi A, Hasebe T, Endo Y, Sasaki S, Konishi M, Sugito M, et al: Primary gastric choriocarcinoma: two case reports and a pooled analysis of 53 cases. Gastric Cancer 2005;8:178-185.

-2 Yoon JH, et al: Primary gastric choriocarcinoma: two case reports and review of the literatures. Cancer Res Treat 2008;40:145-150.

3 Noguchi T, Takeno S, Sato T, Takahashi Y, Uchida Y, Yokoyama S: A patient with primary gastric choriocarcinoma who received a correct preoperative diagnosis and achieved prolonged survival. Gastric Cancer 2002;5:112-117.

4 Shastri A, Daver NG, Hayes TG: Primary gastric chorioadenocarcinoma: a needle in a haystack. Rare Tumors 2011;3:e19.

-5 Liu AY, Chan WY, Ng EK, Zhang X, Li BC, Chow JH, et al: Gastric choriocarcinoma shows characteristics of adenocarcinoma and gestational choriocarcinoma: a comparative genomic hybridization and fluorescence in situ hybridization study. Diagn Mol Pathol 2001;10:161-165.

-6 Bang YJ, et al: Trastuzumab in combination with chemotherapy versus chemotherapy alone for treatment of HER2-positive advanced gastric or gastro-oesophageal junction cancer (ToGA): a phase 3 , open-label, randomised controlled trial. Lancet 2010;376:687-697.

7 Fulop V, Mok SC, Genest DR, Szigetvari I, Cseh I, Berkowitz RS: c-myc, c-erbB-2, c-fms and bcl-2 oncoproteins. Expression in normal placenta, partial and complete mole, and choriocarcinoma. J Reprod Med 1998;43:101-110.

8 Mandoky L, Geczi L, Bodrogi I, Toth J, Csuka O, Kasler M, Bak M: Clinical relevance of HER-2/neu expression in germ-cell testicular tumors. Anticancer Res 2004;24:2219-2224. 